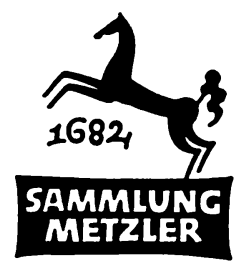

REALIEN ZUR LITERATUR

ABT.: D

LITERATURGESCHICHTE 


\section{Prosa des \\ Expressionismus}

MCMLXXXIV

J. B. METZLERSCHE VERLAGSBUCHHANDLUNG

STUTTGART 
CIP-Kurztitelaufnahme der Deutschen Bibliothek

\section{Krull, Wilhelm:}

Prosa des Expressionismus / Wilhelm Krull. -

Stuttgart: Metzler, 1984.

(Sammlung Metzler; M 210: Abt. D, Literaturgeschichte)

ISBN 978-3-476-10210-2

\section{ISBN 978-3-476-10210-2 ISBN 978-3-476-03914-9 (eBook) DOI 10.1007/978-3-476-03914-9}

(C) 1984 Springer-Verlag GmbH Deutschland

Ursprünglich erschienen bei J. B. Metzlersche Verlagsbuchhandlung und Carl Ernst Poeschel Verlag GmbH in Stuttgart 1984 
I. Vorwort-Erste Umrisse . . . . . . . . . . . . . 1

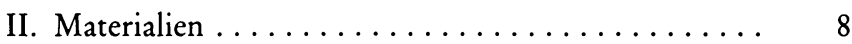

1. Anthologien und Dokumente . . . . . . . . 10

2. Literaturgeschichtliche Arbeiten .......... 13

III. Sprachskepsis, Normen- und Wissenschaftskritik ... 17

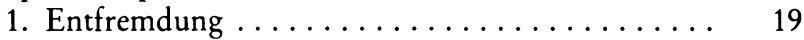

2. Wahnsinn $\ldots \ldots \ldots \ldots \ldots \ldots \ldots \ldots \ldots \ldots \ldots$

IV. Vitalistische und utopische Prosa $\ldots \ldots \ldots \ldots \ldots \ldots \quad 45$

1. Geschichte und Gegenwart . . . . . . . . . 47

2. Utopie ................... 59

V. Aktivistische und gesellschaftskritische Prosa ...... 68

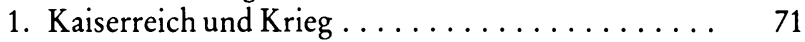

2. Revolution ................. 83

VI. Dadaistische Prosa . . . . . . . . . . . . 92

VII. Ausklänge und Neuanfänge-der moderne Roman . . . 103

VIII. Bibliographische Übersicht - Forschungsstand zu den

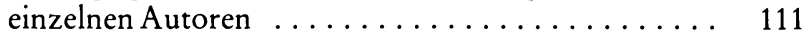

Register ...................... 128 


\section{AbKüRZUNGEN}

DV js Deutsche Vierteljahrsschrift für Literaturwissenschaft und Geistesgeschichte

GRM Germanisch-Romanische Monatsschrift

FAZ Frankfurter Allgemeine Zeitung

MAL Modern Austrian Literature

NDH Neue Deutsche Hefte

NGS New German Studies

OGS Oxford German Studies

Orbis Litt. Orbis Litterarum

Schiller-Jb. Jahrbuch der Deutschen Schillergesellschaft

WB Weimarer Beiträge

WW Wirkendes Wort

ZfGerm Zeitschrift für Germanistik 


\section{VORWORT - ERSTE UMRISSE}

Literarhistorische Studien präsentieren dem heutigen Betrachter ein Bild des Expressionismus, das ihm etwa so verwirrend, vielfältig und bunt erscheinen dürfte wie im Jahre 1911 dem Besucher einer Ausstellung der Berliner Sezession die Gemälde der jungen französischen Künstler, die im Katalog erstmals unter dem Namen »Expressionisten « vorgestellt wurden. Diente diese Bezeichnung damals vor allem der Abgrenzung der neuen von der unmittelbar vorhergehenden, als traditionalistisch abgelehnten Malerei, so werden jetzt eine ganze Anzahl unterschiedlicher, bisweilen sogar gegensätzlicher Phänomene mit dem Begriff Expressionismus verknüpft.

Je nach Forschungsschwerpunkt wird entweder ein weit- oder ein enggefaßter Expressionismusbegriff verwendet; manche möchten ihn gar völlig aufgeben. Einerseits entsteht so der Eindruck, es handele sich beim Expressionismus um eine weit ausgreifende kulturrevolutionäre Bewegung von internationaler Bedeutung, andererseits faßt man zuweilen darunter nur die von lärmender Rhetorik und O-Mensch-Pathos geprägte Literatur des Zeitraums 19091925, die lediglich in Lyrik und Drama adäquate Ausdrucksmöglichkeiten fand. Sehen die einen im Expressionismus die Verkörperung eines radikalen Bruchs mit der vergangenen Kunst, so bestreiten andere gar den Sinn einer Abgrenzung verschiedener >Ismen und verweisen auf die Kontinuität der Moderne bzw. gehen dazu über, Literatur nach sozialgeschichtlichen Gesichtspunkten zu periodisieren.

Solche neueren Versuche, eine Literaturgeschichte des Deutschen Kaiserreichs oder der Weimarer Republik zu schreiben, zeigen jedoch rasch, daß auf eine genauere Darstellung der unterschiedlichen künstlerischen Ansätze nicht verzichtet werden kann und die literarischen Epochenbezeichnungen unter der Hand wieder eingeführt werden. Gleichwohl ergibt sich aus dem Umgang mit den Werken der Schriftsteller dieser Epoche die Schwierigkeit, bei aller notwendigen Differenzierung noch zu einer Definition des Expressionismus zu gelangen; zumal die Autoren auch damals nicht als eine einheitliche Gruppe auftraten. Erst wenn man die Positionen in der Forschung als Akzentuierungen unterschiedlicher Strömungen innerhalb des Expressionismus begreift, läßt sich die Spannweite der Auffassungen als in der Sache selbst begründete Vielfalt der Ansätze erkennen.

Die Formulierung einer kompakten Definition wird auf diese Weise allerdings erschwert, wenn nicht gar unmöglich. 
"Die Aporie des Versuchs, exakt zu bestimmen, was der Expressionismus war, wuchs im Maße des Abstands von diesem Jahrzehnt. Diese Entwicklung hat aber der Expressionismusforschung nur genutzt. Man definierte nicht mehr apodiktisch, sondern suchte Übergänge und Abgrenzungen; man ging einzelnen Aspekten nach." (Rötzer, 16)

Damit rückte in den sechziger Jahren jedoch der individuelle Stil von Autoren wie Benn, Heym, Sternheim und Kafka in den Vordergrund. Der Begriff Expressionismus sank zu einer pejorativ besetzten Sammelbezeichnung für modisch-zeitverhaftete Werke weniger bekannter Autoren herab. Literaturwissenschaftler, die sich darum bemühten, einen Überblick über bestimmte Formentwicklungen oder literaturpolitische Konzeptionen zu geben, schreckten immer öfter davor zurück, den Begriff näher zu bestimmen. Sie benutzten ihn häufig nur noch als »bloßen Namen" (Hohendahl, 12) für das entsprechende Jahrzehnt bzw. die zwischen 1909 und 1925 entstandenen Werke. Als man in den siebziger Jahren begann, das gesamte Spektrum unterschiedlicher Motivkomplexe und Schreibhaltungen aufzufächern, ließ sich das gesichtete Material kaum noch unter einem Oberbegriff zusammenfassen.

"Avantgardistischem Drang zu ästhetischer Revolution, dem Primat formaler Innovation bei den einen Autoren widerspricht bei anderen totale Gleichgültigkeit hinsichtlich Formfragen, ja ein explizites Negieren ihrer Bedeutung. Für eine so knappe Spanne - die Jahre 1909 bis etwa 1925, anderthalb Dezennien nur - ließen sich schwerlich größere Unterschiede der Form, eine breitere Skala von >Individualstilen finden. " (Rothe, 12)

Die Bandbreite der Formentwürfe schlägt sich in der Forschungsliteratur zur Prosa des Expressionismus in der Tendenz zur Auskristallisierung exemplarischer Pole nieder. Sie macht sich an einzelnen Schriftstellern (z. B. Einstein vs. Döblin, Sternheim vs. Meyrink), an Gruppenbildungen (Aktions- vs. Sturmkreis, Berliner vs. Münchner Autoren) oder an Literaturkonzeptionen (Vitalismus vs. Aktivismus, Wortkunst vs. Dadaismus etc.) fest und verabsolutiert nicht selten die jeweilige Gegenüberstellung zur entscheidenden Auseinandersetzung im Expressionismus überhaupt. Als besonders problematisch erweist sich ein solches Verfahren, wenn die beiden Positionen eher miteinander verwandte als diametral entgegengesetzte Punkte der expressionistischen Prosa markieren wie z.B. in Walter Sokels Vorschlag, an Hand der Erzähltheorien Döblins und Einsteins zwischen einer expressionistisch-subjektivierenden und einer naturalistisch-objektivierenden Richtung innerhalb des Expressionismus zu unterscheiden. 
"Während Döblin das Bewußtsein des Schreibenden völlig in dessen Produkt, den dargestellten Vorgang, verschwinden und es nur mittelbar in seiner Wirkung, dem Werk, erscheinen läßt, manifestiert sich in der Einsteinschen Richtung dieses Bewußtsein entweder unmittelbar in den Reflexionen des Erzählers - daher die Bevorzugung der Ichform - oder mittelbar in den Reflexionen einer Figur, die das Erzählte dauernd kommentiert und bedenkt, ja deren Reflexionen an die Stelle von Darstellung treten. " (Sokel, 157)

Obwohl es sich hier um eine wichtige Beobachtung handelt und es zunächst verlockend erscheinen mag, die expressionistische Prosa nach Erzählstrukturen und -perspektiven zu typologisieren, sind die Schwächen dieser Konstruktion kaum zu übersehen: Sie verengt nicht nur die Vielfalt der Ansätze in der expressionistischen Prosa auf zwei formal ausgerichtete Konzepte (deren grundlegende Gemeinsamkeit - die Ablehnung des psychologischen Realismus - auch Sokel einräumt), sondern verstellt sich auch die Möglichkeit, die literarische Praxis expressionistischer Schriftsteller als Resultat der Bearbeitung verschiedener Stoffe unter den Bedingungen einer besonderen historischen Denkform zu begreifen. Die Problematik seines Vorgehens - insbesondere die Austauschbarkeit des entscheidenden Kriteriums - scheint Sokel selbst einzugestehen, wenn er nach erzähltechnischen Gesichtspunkten Autoren wie Kubin, Meyrink, Müller und Kafka zum Expressionismus zählt, gleich danach aber schreibt:

"Sprachlich läßt sich übrigens keiner dieser vier österreichischen - Prager und Wiener - Allegoriker dem Expressionismus zuschreiben, ebensowenig wie Musil oder Heinrich Mann sprachlich als Expressionisten anzusehen sind. Denn wenn es überhaupt etwas gibt, was die expressionistische Prosa sprachlich kennzeichnet, dann die folgenden drei Faktoren: Parataxe, Ellipsis und schließlich syntaktische Satzverzerrung." (Sokel, 165)

Die Konzentration auf einzelne Aspekte ist für Spezialuntersuchungen zweifellos sinnvoll und notwendig zur Erhellung der veränderten Erkenntnis- und Gestaltungsabsichten expressionistischer Autoren gegenüber ihren Vorgängern. Im Rahmen einer übergreifenden Darstellung würde sie zwar die Grenzziehung erleichtern, aber auch das Blickfeld so sehr verengen, daß es kaum möglich wäre, die Bandbreite der Ausdrucksmöglichkeiten innerhalb der Prosa dieses Zeitraums und die bedeutendsten Entwicklungslinien aufzuzeigen. Mit einer Ausfaltung des gesamten Spektrums sprachlicher und erzähltechnischer Innovationen soll deshalb hier versucht werden, ein Bild von den verschiedenen Ausdruckstendenzen und deren inneren Zusammenhängen zu zeichnen, das historische und systematische Momente gleichermaßen berücksichtigt und den Blick auf parallele Erscheinungen bzw. unterschiedliche Bearbeitungen des 
gleichen Themas freigibt. Bei einer solchen Vorgehensweise ist es zwar unvermeidlich, daß in Einzelfällen die Werke eines Schriftstellers verschiedenen Strömungen innerhalb des Expressionismus zugeordnet werden; dies läßt sich jedoch oftmals - wie z.B. im Fall René Schickeles, der sich vom vitalistischen zum aktivistischen $\mathrm{Au}-$ tor wandelte - aus den Brüchen in der künstlerischen Entwicklung erklären bzw. damit rechtfertigen, daß ein bestimmtes Thema unter veränderten Bedingungen wieder aufgegriffen wird.

Die Unterscheidung von vier Haupttendenzen innerhalb des Expressionismus deckt sich ungefähr mit dessen Entwicklungsgeschichte. Sie läßt sich etwa so skizzieren: In den Jahren vor dem Ersten Weltkrieg stand die normen-, sprach- und wissenschaftskritische Prosa mit ihrer Thematisierung des Sprach- und Realitätszerfalls, der Reflexion auf die Darstellungsmöglichkeiten sowie die Schwierigkeiten ästhetischer Sinnkonstitution im Zentrum des Interesses. Parallel dazu entwickelte sich bereits die vitalistische und utopische Prosa. Während aber Wirklichkeitszerfall und Erkenntnisskepsis in den letzten Kriegsjahren - vorübergehend - in den Hintergrund traten, erschienen vitalistische und utopische Texte kontinuierlich bis in die zwanziger Jahre hinein. Der Krieg bedeutete jedoch auch für diese Autoren, auf die Nietzsches Lebensphilosophie besonders prägend gewirkt hatte, eine Zäsur: Viele Metaphern wurden aufs grausamste von der Wirklichkeit überholt. Die Erfahrungen auf den Schlachtfeldern veranlaßten viele Schriftsteller zu einer Revision ihrer Vorstellungen von einem kraftvollen Leben. Der gesellschaftskritisch und pazifistisch orientierte Aktivismus wurde im Laufe des Krieges zur dominierenden Bewegung. Auf deren politisch-moralisches Literaturverständnis reagierten dann die dadaistischen Schriftsteller mit höhnischen Angriffen. Bei einigen von ihnen ist wiederum ein deutlicher Rückbezug auf die erkenntniskritische Prosa der Vorkriegszeit (z. B. Hugo Balls auf Carl Einsteins "Bebuquin «) nachweisbar.

Trotz divergenter Erzählansätze lassen sich eine Reihe von Gemeinsamkeiten und Entsprechungen herausarbeiten, die dazu berechtigen, von der Prosa des Expressionismus als einer literaturgeschichtlichen Einheit zu sprechen. Ihre Bedeutung für die Entwicklung des modernen Romans liegt vor allem in der Erschließung neuer Aussage- und Wirkungsmöglichkeiten. Die Aufnahme dramatischer und essayistischer Formen in den Roman, das allenthalben sichtbare Bedürfnis der Autoren, ihr eigenes Romanschaffen in poetologischen Erörterungen zu legitimieren, indizieren eine Krise des traditionellen, der persönlichen Erfahrung gewissen Erzählens, die in der Ohnmacht des Individuums angesichts der Weltkriegs- 
und Inflationszeit ihre soziale Grundlage hat. Die expressionistischen Romane, Erzählungen, Prosaskizzen, Essays, Pamphlete und Manifeste bilden gewissermaßen das Experimentierstadium, ohne das die heute als Paradigmen des modernen Romans anerkannten Bücher wie Musils "Mann ohne Eigenschaften «, Döblins "Berlin Alexanderplatz " und Brochs "Schlafwandler " kaum in dieser Weise möglich gewesen wären.

Obgleich durch die Veröffentlichungen der letzten Jahre die Prosa etwas von dem Ruf verloren haben dürfte, ein »Stiefkind der Expressionismusforschung " (Knapp, 91) zu sein, ist es noch immer schwierig, bibliographische Angaben über die Arbeiten zu einzelnen, insbesondere den weniger bekannten Autoren zu finden. Der Band schließt deshalb mit einer Übersicht über den jeweiligen Forschungsstand zu den einzelnen Schriftstellern.

\section{Zitierte Literatur:}

Hans Gerd Rötzer (Hg.): Begriffsbestimmung des literarischen Expressionismus. Darmstadt 1976.

Peter Uwe Hohendabl: Das Bild der bürgerlichen Welt im expressionistischen Drama. Heidelberg 1967.

Wolfgang Rothe: Der Expressionismus. Theologische, soziologische und anthropologische Aspekte einer Literatur. Frankfurt a. M. 1977.

Walter H. Sokel: Die Prosa des Expressionismus. In: Wolfgang Rothe (Hg.): Expressionismus als Literatur. Gesammelte Studien. Bern und München 1969. S. 153-170.

Gerhard P. Knapp: Die Literatur des deutschen Expressionismus. Einführung-Bestandsaufnahme - Kritik. München 1979.

\section{Weitere Literatur:}

Armin Arnold: Die Literatur des Expressionismus. Sprachliche und thematische Quellen. Stuttgart u.a. 1966.

Gertrud Bauer/Karl Eugene Webb (Hg.): Expressionism Reconsidered. Relationship and Affinities. München 1979.

Benjamin Bennett et al. (Hg.): Probleme der Moderne. Studien zur deutschen Literatur von Nietzsche bis Brecht. Festschrift für Walter H. Sokel. Tübingen 1982.

Jan Berg et al.: Sozialgeschichte der deutschen Literatur von 1918 bis zur Gegenwart. Frankfurt a. M. 1981.

Richard Brinkmann: Expressionismus. Internationale Forschung zu einem internationalen Phänomen. Sonderband der DVjs. Stuttgart 1980.

Gerald Chaple/Hans H. Schulte (Hg.): The Turn of the Century. German Literature and Art, 1890-1915. The McMaster Colloquium on German Literature. Bonn 1981.

Brigitte Dörrlmann et al.: Klassiker heute. Die Zeit des Expressionismus. 
Erste Begegnung mit Georg Heym, Georg Trakl, Gottfried Benn, Johannes R. Becher, Georg Kaiser, Alfred Döblin, Else Lasker-Schüler, Ernst Toller. Frankfurt a. M. 1982.

Günther Erken: Der Expressionismus. Anreger, Herausgeber, Verleger. In: Hermann Kunisch (Hg.): Handbuch der deutschen Gegenwartsliteratur. Bd. 2. 2. Aufl. München 1970. S. 335-364.

Christoph Eykman: Denk- und Stilformen des Expressionismus. München 1974.

Peter Gay: Freud, Jews and Other Germans. Masters and Victims in Modernist Culture. New York 1978.

Richard Hamann/Jost Hermand: Epochen deutscher Kultur von 1870 bis zur Gegenwart. Bd. 5: Expressionismus. Frankfurt a. M. 1977.

Jost Hermand: Stile, Ismen, Etiketten. Zur Periodisierung der modernen Kunst. Wiesbaden 1978.

Bruno Hillebrand (Hg.): Nietzsche und die deutsche Literatur. Bd. 1: Texte zur Nietzsche-Rezeption 1873-1963. Bd. 2: Forschungsergebnisse. Tübingen 1978.

Klaus Günter Just: Von der Gründerzeit bis zur Gegenwart. Geschichte der deutschen Literatur seit 1871. Bern und München 1973.

Hans Kaufmann: Krisen und Wandlungen der deutschen Literatur von Wedekind bis Feuchtwanger. Fünfzehn Vorlesungen. 3. Aufl. Berlin und Weimar 1976.

Hans-Georg Kemper: Vom Expressionismus zum Dadaismus. Eine Einführung in die dadaistische Literatur. Kronberg/Ts. 1974.

Helmut Kreutzer: Zur Periodisierung der 'modernen deutschen Literatur. In: Basis. Jahrbuch für deutsche Gegenwartsliteratur 2 (1971). S. 7-32.

Eberhard Lämmert (Hg.): Erzählforschung. Ein Symposion. Stuttgart 1982.

Gunter Martens: Vitalismus und Expressionismus. Ein Beitrag zur Genese und Deutung expressionistischer Stilstrukturen und Motive. Stuttgart 1971.

Horst Meixner/Silvio Vietta (Hg.): Expressionismus. Sozialer Wandel und künstlerische Erfahrung. Mannheimer Colloquium. München 1981.

Wolfgang Paulsen: Deutsche Literatur des Expressionismus. Bern u.a. 1983. Wolfgang Paulsen: Expressionismus und Aktivismus. Eine typologische Untersuchung. Bonn und Leipzig 1935.

Wolfgang Paulsen (Hg.): Aspekte des Expressionismus. Periodisierung, Stil, Gedankenwelt. Die Vorträge des Ersten Kolloquiums in Amherst/Mass. Heidelberg 1968.

Geoffrey C. Perkins: Contemporary Theory of Expressionism. Bern und Frankfurt a. M. 1974.

Geoffrey C. Perkins: Expressionismus. Eine Bibliographie zeitgenössischer Dokumente 1910-1925. Zürich 1971.

Wolfdietrich Rasch: Zur deutschen Literatur seit der Jahrhundertwende. Stuttgart 1967.

Lionel Richard (Hg.): Lexikon des Expressionismus. Gütersloh o.J. (1979). Wolfgang Rothe: Tänzer und Täter. Gestalten des Expressionismus. Frankfurt a.M. 1979. 
Erhard Schütz u.a.: Einführung in die deutsche Literatur des 20. Jahrhunderts. Bd. 1: Kaiserreich. Opladen 1977.

Richard W. Sheppard: The Expressionist Cabaret GNU (1911-1914): An Analysis and Documentation. In: DVjs. 56 (1982). S. 431-446.

Frank Trommler ( $\mathrm{Hg}$.): Jahrhundertwende: Vom Naturalismus zum Expressionismus 1880-1918. Reinbek bei Hamburg 1982.

Silvio Vietta/Hans-Georg Kemper: Expressionismus. München 1975.

Viktor Žmegač (Hg.): Deutsche Literatur der Jahrhundertwende. Königstein/Ts. 1981. 